\title{
ON THE MODULI SPACES OF COMMUTING ELEMENTS IN THE PROJECTIVE UNITARY GROUPS
}

\author{
ALEJANDRO ADEM AND MAN CHUEN CHENG
}

\begin{abstract}
We provide descriptions for the moduli spaces $\operatorname{Rep}(\Gamma, P U(m))$, where $\Gamma$ is any finitely generated abelian group and $P U(m)$ is the group of $m \times m$ projective unitary matrices. As an application we show that for any connected CW-complex $X$ with $\pi_{1}(X) \cong$ $\mathbb{Z}^{n}$, the natural map $\pi_{0}\left(\operatorname{Rep}\left(\pi_{1}(X), P U(m)\right)\right) \rightarrow[X, B P U(m)]$ is injective, hence providing a complete enumeration of the isomorphism classes of flat principal $P U(m)$-bundles over $X$.
\end{abstract}

\section{INTRODUCTION}

The space of ordered commuting $n$-tuples in a compact, connected Lie group $G$ is by definition the subspace $\operatorname{Hom}\left(\mathbb{Z}^{n}, G\right) \subset G^{n}$ (see [4] for background and basic properties). Its orbit space under conjugation, denoted $\operatorname{Rep}\left(\mathbb{Z}^{n}, G\right)$, can be identified with the moduli space of isomorphism classes of flat connections on principal $G$-bundles over the $n$-dimensional torus $\mathbb{T}^{n}$. In the case when all of the maximal abelian subgroups of $G$ are path connected, it can be shown that this moduli space has a single connected component, corresponding to the identity element $(1, \ldots, 1) \in G^{n}$ (see [4], Proposition 2.3). For example, $\operatorname{Rep}\left(\mathbb{Z}^{n}, U(m)\right) \cong S P^{m}\left(\mathbb{T}^{n}\right)$, the $m$-fold symmetric product of the $n$-torus. However by a result due to Borel (see [6], page 216), for any prime number $p$ the fundamental group of $G$ has $p$-torsion if and only if there exists a rank two elementary abelian $p$-subgroup of $G$ which is not a subgroup of any torus. In this case $\operatorname{Rep}\left(\mathbb{Z}^{n}, G\right)$ fails to be path-connected for all $n \geq 2$ and determining the number and exact structure of the components can be fairly complicated. Borel also shows that $H^{*}(G, \mathbb{Z})$ has $p$-torsion if and only if there exists a subgroup of the form $(\mathbb{Z} / p \mathbb{Z})^{3} \subset G$ which is not contained in any torus. This can be used to show for example that $\operatorname{Rep}\left(\mathbb{Z}^{3}, \operatorname{Spin}(7)\right)$ is not path connected, even though $\operatorname{Spin}(7)$ is simply connected.

In this note we consider the case when $G=P U(m)$, the group of $m \times m$ projective unitary matrices, which has fundamental group isomorphic to $\mathbb{Z} / m \mathbb{Z}$ and

$$
H^{2}(B P U(m), \mathbb{Z} / m \mathbb{Z}) \cong \mathbb{Z} / m \mathbb{Z}
$$

has a canonical generator $\nu$ of order $m$, corresponding to the central extension

$$
1 \rightarrow \mathbb{Z} / m \mathbb{Z} \rightarrow S U(m) \rightarrow P U(m) \rightarrow 1 .
$$

Given a homomorphism $h: \mathbb{Z}^{n} \rightarrow P U(m)$, one can associate to it the cohomology class $\alpha=h^{*}(\nu) \in H^{2}\left(\mathbb{Z}^{n}, \mathbb{Z} / m \mathbb{Z}\right)$. For our purposes it's convenient to identify $H^{2}\left(\mathbb{Z}^{n}, \mathbb{Z} / m \mathbb{Z}\right)$ with the set $T(n, \mathbb{Z} / m \mathbb{Z})$ of $n \times n$ skew-symmetric matrices over $\mathbb{Z} / m \mathbb{Z}$ : given a basis $z_{1}, \ldots, z_{n}$ for $\mathbb{Z}^{n}$, then $D=\left(d_{i j}\right) \in T(n, \mathbb{Z} / m \mathbb{Z})$ corresponds to $\sum_{i<j} d_{i j} z_{i}^{*} z_{j}^{*}$. 
Now given a skew-symmetric $n \times n$ matrix $D$ over $\mathbb{Z} / m \mathbb{Z}$ representing $\alpha$, we define $\sigma(\alpha)=\sigma(D)=\sqrt{|R(D)|}$, where $R(D) \subset(\mathbb{Z} / m \mathbb{Z})^{n}$ is the row space of $D$ (see [1], Definition 2). Alternatively, for $\alpha \in H^{2}\left(\mathbb{Z}^{n}, \mathbb{Z} / m \mathbb{Z}\right)$, by [8], Proposition 4.1 we can find a basis $g_{1}, \ldots, g_{n}$ of $\mathbb{Z}^{n}$ such that $\alpha=c_{1} g_{1}^{*} g_{2}^{*}+\cdots+c_{r} g_{2 r-1}^{*} g_{2 r}^{*}$ where $2 r \leq n, c_{1}, \ldots, c_{r} \in \mathbb{Z} / m \mathbb{Z}$ and $\left|c_{r}\right||| c_{r-1}|| \ldots\left|c_{1}\right|$. Using this basis it follows that $\sigma(\alpha)=\prod_{i=1}^{r}\left|c_{i}\right|$.

For a topological group $G$, let $\widehat{S P^{n}}(G)$ denote the reduced $n$-fold symmetric product of $G$, defined as the quotient $S P^{n}(G) / G$, where $G$ acts by translation on each unordered coordinate. Then our main result can be stated as follows:

Theorem A. For all $m, n \geq 1$ there are homeomorphisms

$$
\operatorname{Rep}\left(\mathbb{Z}^{n}, P U(m)\right) \cong \coprod_{\substack{D \in T(n, \mathbb{Z} / m \mathbb{Z}) \\ \sigma(D) \mid m}} \widehat{S P^{\frac{m}{\sigma(D)}}}\left(\mathbb{T}^{n} / R(D)\right)
$$

Note that $\mathbb{T}^{n} / R(D) \cong \mathbb{T}^{n}$, so our formula expresses the moduli space as a disjoint union of reduced symmetric products of the $n$-torus. The number of path-connected components of $\operatorname{Rep}\left(\mathbb{Z}^{n}, P U(m)\right)$ is equal to $N(n, m)=\mid\{D \in T(n, \mathbb{Z} / m \mathbb{Z}) \mid \sigma(D)$ divides $m\} \mid$, a rather intricate number that has been computed in Adem-Cheng (see [1], Corollary 3.9). We also show how to apply our methods to provide a description of $\operatorname{Rep}(\Gamma, P U(m))$ for any finitely generated abelian group $\Gamma$ (see Theorem [2.11). Note that by the results in [5], for these groups there is a homotopy equivalence $\operatorname{Rep}(\Gamma, P G L(m, \mathbb{C})) \simeq \operatorname{Rep}(\Gamma, P U(m))$.

Recall that for $G$ a topological group and $X$ a CW-complex, the homotopy classes of maps from $X$ to $B G$, denoted $[X, B G]$, classify isomorphism classes of principal $G$ bundles over $X$. Representations play a key in this through the theory of flat bundles; in our setting the key connection is via the induced map on components

$$
\Psi_{\mathbb{T}^{n}}^{P U(m)}: \pi_{0}\left(\operatorname{Rep}\left(\mathbb{Z}^{n}, P U(m)\right)\right) \rightarrow\left[\mathbb{T}^{n}, B P U(m)\right] .
$$

Taking composition with the classifying map $c_{X}: X \rightarrow B \pi_{1}(X)$, we obtain the following general result

Theorem B. Let $X$ denote a connected $C W$-complex with $\pi_{1}(X)=\mathbb{Z}^{n}$; then the map

$$
\Psi_{X}^{P U(m)}: \pi_{0}\left(\operatorname{Rep}\left(\pi_{1}(X), P U(m)\right)\right) \rightarrow[X, B P U(m)]
$$

is injective for all $n, m \geq 1$ and so there are $N(n, m)$ distinct isomorphism classes of flat $P U(m)$-bundles on $X$.

Regarding surjectivity, we obtain that for all $m \geq 2$,

$$
\Psi_{\mathbb{T}^{n}}^{P U(m)}: \pi_{0}\left(\operatorname{Rep}\left(\mathbb{Z}^{n}, P U(m)\right)\right) \rightarrow\left[\mathbb{T}^{n}, B P U(m)\right]
$$

is surjective if and only if $n \leq 3$ (Proposition 3.2). It follows that there exists a principal $P U(m)$-bundle on the $n$-torus $\mathbb{T}^{n}$ which does not admit a flat structure if and only if $n \geq 4$. Here we apply the results in [9], which provide a classification of principal $P U(m)$-bundles over low-dimensional complexes.

The results in this paper can be viewed as an application and refinement of the analysis carried out in our previous work [1], where we described the space $B_{n}(U(m))$ of almost commuting $n$-tuples in $U(m)$. 
ON THE MODULI SPACES OF COMMUTING ELEMENTS IN THE PROJECTIVE UNITARY GROUPS 3

\section{Projective Representations and Almost Commuting Elements}

In this section we will apply the methods from [1] to give a description of $\operatorname{Rep}\left(\mathbb{Z}^{n}, P U(m)\right)$. The almost commuting elements will play a crucial role.

Definition 2.1. We define $B_{n}(G)$, the almost commuting elements in a Lie group $G$, as the set of all ordered $n$-tuples $\left(A_{1}, A_{2}, \ldots, A_{n}\right) \in G^{n}$ such that the commutators $\left[A_{i}, A_{j}\right] \in Z(G)$, the centre of $G$, for all $1 \leq i, j \leq n$.

Let $F_{n}$ denote the free group on $n$ generators $a_{1}, \ldots a_{n}$. We will identify a map from $F_{n}$ to $G$ with the $n$-tuple of images of these generators in $G$. Suppose that $f: F_{n} \rightarrow U(m)$ is in $B_{n}(U(m))$. For any $u, v \in F_{n},[f(u), f(v)]=\gamma I_{m}$ for some $m$-th root of unity $\gamma$, as the determinant of a commutator of invertible matrices is equal to one. The exponential function $z \mapsto e^{2 \pi \sqrt{-1} z}$ establishes a group isomorphism between $\mathbb{R} / \mathbb{Z}$ and $\mathbb{S}^{1} \subset \mathbb{C}$ with inverse $w \mapsto \frac{1}{2 \pi \sqrt{-1}} \log w$. The multiplicative groups of $m$-th roots of unity and all roots of unity correspond to the subgroup $\mathbb{Z}\left[\frac{1}{m}\right] / \mathbb{Z} \cong \mathbb{Z} / m \mathbb{Z}$ and $\mathbb{Q} / \mathbb{Z}$ of $\mathbb{R} / \mathbb{Z}$ respectively under this isomorphism. We will be using this identification from now on. Hence there is a map $F_{n} \times F_{n} \rightarrow \mathbb{Z} / m \mathbb{Z} \subset \mathbb{Q} / \mathbb{Z}$ defined by $(u, v) \mapsto \frac{1}{2 \pi \sqrt{-1}} \log \gamma$. Since $f\left(\left[F_{n}, F_{n}\right]\right) \subset Z(U(m))$, the map factors through the abelianization of $F_{n} \times F_{n}$ and thus gives rise to a $\mathbb{Z} / \mathrm{m} \mathbb{Z}$-valued skew-symmetric bilinear form $\omega_{f}: \mathbb{Z}^{n} \times \mathbb{Z}^{n} \rightarrow \mathbb{Z} / m \mathbb{Z}$.

Define a map

$$
\rho: B_{n}(U(m)) \rightarrow T(n, \mathbb{Z} / m \mathbb{Z})
$$

by $\rho(f)=\left(d_{i j}\right)$, where $\left[f\left(a_{i}\right), f\left(a_{j}\right)\right]=e^{2 d_{i j} \pi \sqrt{-1}} I_{m}$. For $D \in T(n, \mathbb{Z} / m \mathbb{Z})$, let $B_{n}(U(m))_{D}=$ $\rho^{-1}(D)$. For $f \in B_{n}(U(m))_{D}$, the ordered $n$-tuple $\left(f\left(a_{1}\right), \ldots, f\left(a_{n}\right)\right)$ is said to be $D$ commuting. Note that $\rho(f)$ is the skew-symmetric matrix associated to the bilinear form $\omega_{f}$.

Definition 2.2. For any $n \times n$ matrix $D \in M_{n \times n}(\mathbb{Z} / m \mathbb{Z})$, the row space $R(D)$ is the submodule of $(\mathbb{Z} / m \mathbb{Z})^{n}$ generated by the rows of $D$ over $\mathbb{Z}$. Let $R_{i}(D) \subset \mathbb{Z} / m \mathbb{Z}$ be the image of $R(D)$ under the projection onto the $i$-th factor. Let $r_{i}(D)=\left|R_{i}(D)\right|$ for $i=1, \ldots, n$ and $\sigma(D)=\sqrt{|R(D)|}$.

We recall the structure of the almost commuting $n$-tuples in $U(m)$, established in [1], Corollary 3.6.

Proposition 2.3. For $D \in T(n, \mathbb{Z} / m \mathbb{Z})$, the space $B_{n}(U(m))_{D}$ is non-empty and path connected if $\sigma(D)$ divides $m$, and is empty otherwise. The space $B_{n}(U(m))$ can be expressed as a disjoint union of path connected components

$$
B_{n}(U(m))=\coprod_{\substack{D \in T(n, \mathbb{Z} / m \mathbb{Z}) \\ \sigma(D) \mid m}} B_{n}\left((U(m))_{D} .\right.
$$

We begin our analysis by focusing on the basic case when $m=\sigma(D)$. Let $0 \leq t \leq n / 2$ and $d_{1}, d_{2}, \ldots, d_{t} \neq 0 \in \mathbb{Z} / m \mathbb{Z}$. Define $D_{n}\left(d_{1}, d_{2}, \ldots d_{t}\right)=\left(d_{i j}\right) \in T(n, \mathbb{Z} / m \mathbb{Z})$ be the 
skew-symmetric matrix with

$$
d_{i j}= \begin{cases}d_{k} & \text { if }(i, j)=(k+t, k), 1 \leq k \leq t \\ -d_{k} & \text { if }(i, j)=(k, k+t), 1 \leq k \leq t \\ 0 & \text { otherwise }\end{cases}
$$

Lemma 2.4. Suppose $D \in T(n, \mathbb{Z} / m \mathbb{Z}), m=\sigma(D)$. Let $\left(A_{1}, \ldots A_{n}\right) \in B_{n}(U(m))_{D}$. If $B \in U(m)$ commutes with $A_{i}$ for all $i=1, \ldots, n$, then $B$ is a scalar matrix.

Proof. By [8, Proposition 4.1], there exists $Q=\left(q_{i j}\right) \in G L(n, \mathbb{Z})$ such that $Q^{T} D Q=D^{\prime}=$ $D_{n}\left(d_{1}, \ldots, d_{t}\right)$. Define $D^{\prime \prime}=D_{n+1}\left(d_{1}, \ldots, d_{t}\right)$ and $A_{j}^{\prime}=A_{1}^{q_{1 j}} A_{2}^{q_{2 j}} \ldots A_{n}^{q_{n j}}$ for $j=1, \ldots, n$. Then $\sigma\left(D^{\prime \prime}\right)=m$ and $\left(A_{1}^{\prime}, \ldots A_{n}^{\prime}, B\right) \in B_{n+1}(U(m))_{D^{\prime \prime}}$. By [1, theorem 3.3], there exists an orthonormal basis of $\mathbb{C}^{m}$ consisting of eigenvectors of $B$ corresponding to a common eigenvalue. Hence, $B$ is a scalar matrix.

Lemma 2.5. Suppose $D \in T(n, \mathbb{Z} / m \mathbb{Z})$ and $m=\sigma(D)$. Let $\left(A_{1}, \ldots A_{n}\right) \in B_{n}(U(m))_{D}$ and $\omega=e^{2 \pi \sqrt{-1} / r_{j}(D)}$. If $\lambda$ is an eigenvalue of $A_{j}$, then $\omega^{q} \lambda$, where $q=0, \ldots, r_{j}(D)-1$, are all the distinct eigenvalues of $A_{j}$.

Proof. Let $D=\left(d_{i j}\right)$. There exists $a_{1}, \ldots, a_{n} \in \mathbb{Z}$ such that $a_{1} d_{1 j}+\ldots+a_{n} d_{n j}=\left[-\frac{1}{r_{j}(D)}\right]$. Let $B=A_{1}^{a_{1}} \ldots A_{n}^{a_{n}}$. Then $B A_{j}=\omega^{-1} A_{j} B$. If $v$ is an eigenvector of $A_{j}$ corresponding to eigenvalue $\lambda$, then $A_{j} B v=\omega B A_{j} v=\omega B(\lambda v)=\omega \lambda B v$ and so $\omega \lambda$ is also an eigenvalue of $A_{j}$. Inductively $\omega^{q} \lambda$ is also an eigenvalue of $A_{j}$ for any $q$. On the other hand, $A_{j}^{r_{j}(D)}$ commutes with $A_{1}, \ldots, A_{n}$. By lemma 2.4, $A_{j}^{r_{j}(D)}$ is a scalar matrix. Since $\lambda$ is an eigenvalue of $A_{j}$, $A_{j}^{r_{j}(D)}=\lambda^{r_{j}(D)} I_{m}$ and so any eigenvalue of $A_{j}$ is of the form $\omega^{q} \lambda$.

In the situation of lemma 2.5, take an eigenvalue $\lambda_{j}$ of $A_{j}$. Define

$$
c_{j}=\frac{1}{2 \pi \sqrt{-1}} \log \lambda_{j} \in \mathbb{R} /\left\langle\frac{1}{r_{j}(D)}\right\rangle \cong \mathbb{S}^{1} / R_{j}(D) .
$$

This element $c_{j}$ is independent of the choice of $\lambda_{j}$.

Theorem 2.6. Let $m=\sigma(D)$. There exists a $\mathbb{T}^{n}$-equivariant homeomorphism

$$
B_{n}(U(m))_{D} / U(m) \cong \mathbb{T}^{n} / R(D)
$$

such that its composition with the quotient map

$$
B_{n}(U(m))_{D} \rightarrow B_{n}(U(m))_{D} / U(m) \cong \mathbb{T}^{n} / R(D) \rightarrow \prod_{j=1}^{n} \mathbb{S}^{1} / R_{j}(D)
$$

sends $\left(A_{1}, \ldots A_{n}\right)$ to $\left(c_{1}, \ldots c_{n}\right)$.

Proof. If $D$ is of the special form $D=D_{n}\left(d_{1}, \ldots, d_{t}\right)$, the theorem follows easily from the proof of theorem 3.4 in [1]. In the general case, let $\left(C_{1}, \ldots C_{n}\right) \in B_{n}(U(m))_{D}$. By multiplying by scalars if necessary, we assume that 1 is an eigenvalue of each $C_{i}$. The map $f: \mathbb{T}^{n} \rightarrow B_{n}(U(m))_{D}$ with $f\left(\theta_{1}, \ldots, \theta_{n}\right)=\left(\theta_{1} C_{1}, \ldots, \theta_{n} C_{n}\right)$ is $\mathbb{T}^{n}$-equivariant. Also, there exists $Q=\left(q_{i j}\right) \in G L(n, \mathbb{Z})$ such that $Q^{T} D Q=D^{\prime}=D_{n}\left(d_{1}, \ldots, d_{t}\right)$. Define $\phi: \mathbb{T}^{n} \rightarrow \mathbb{T}^{n}$ by $\phi\left(\theta_{1}, \ldots, \theta_{n}\right)=\left(\theta_{1}^{\prime}, \ldots, \theta_{n}^{\prime}\right)$ and $g: B_{n}(U(m))_{D} \rightarrow B_{n}(U(m))_{D^{\prime}}$ by $g\left(A_{1}, \ldots, A_{n}\right)=\left(A_{1}^{\prime}, \ldots, A_{n}^{\prime}\right)$, where $\theta_{j}^{\prime}=\theta_{1}^{q_{1 j}} \ldots . \theta_{n}^{q_{n j}}$ and $A_{j}^{\prime}=A_{1}^{q_{1 j}} A_{2}^{q_{2 j}} \ldots A_{n}^{q_{n j}}$ for 
ON THE MODULI SPACES OF COMMUTING ELEMENTS IN THE PROJECTIVE UNITARY GROUPS 5 $j=1, \ldots, n$. Note that $g$ is a $\phi$-equivariant and $U(m)$-equivariant homeomorphism. Using the result for the special case $D^{\prime}$, it can be deduced that the composition

$$
\mathbb{T}^{n} / R\left(D^{\prime}\right) \stackrel{\bar{\phi}^{-1}}{\cong} \mathbb{T}^{n} / R(D) \stackrel{\bar{f}}{\rightarrow} B_{n}(U(m))_{D} / U(m) \stackrel{\bar{g}}{\cong} B_{n}(U(m))_{D^{\prime}} / U(m)
$$

is a homeomorphism and so is $\bar{f}$. It is clear that $\bar{f}^{-1}$ has the desired properties.

Note that $R(D)$ is a finite subgroup of $\mathbb{T}^{n}$, acting by translation; thus $\mathbb{T}^{n} \rightarrow \mathbb{T}^{n} / R(D)$ is a covering space and $\mathbb{T}^{n} / R(D)$ is homeomorphic to $\mathbb{T}^{n}$.

For the general case $m=l \cdot \sigma(D)$, we recall that from [1], Corollary 3.10 that

$$
B_{n}(U(m))_{D} / U(m) \cong\left(B_{n}(\sigma(D))_{D} / U(\sigma(D))\right)^{l} / \Sigma_{l}=S P^{l}\left(B_{n}(\sigma(D))_{D} / U(\sigma(D))\right) .
$$

Hence, we obtain that

Theorem 2.7. The moduli space of ordered almost commuting $n$-tuples in $U(m)$ can be expressed as a disjoint union of symmetric products of the $n$-torus $\mathbb{T}^{n}$ :

$$
B_{n}(U(m)) / U(m) \cong \coprod_{\substack{D \in T(n, \mathbb{Z} / m \mathbb{Z}) \\ \sigma(D) \mid m}} S P^{\frac{m}{\sigma(D)}}\left(\mathbb{T}^{n} / R(D)\right)
$$

Now we apply the following result.

Lemma 2.8 ([2], Lemma 2.3). The projection map $U(m) \rightarrow P U(m)$ induces a $U(m)-$ equivariant, $\mathbb{T}^{n}$-principal bundle

$$
B_{n}(U(m)) \rightarrow \operatorname{Hom}\left(\mathbb{Z}^{n}, P U(m)\right)
$$

which gives rise to a homeomorphism

$$
\mathbb{T}^{n} \backslash B_{n}(U(m)) / U(m) \cong \operatorname{Rep}\left(\mathbb{Z}^{n}, P U(m)\right) .
$$

In particular it induces a bijection between $\pi_{0}\left(B_{n}(U(m)) / U(m)\right)$ and $\pi_{0}\left(\operatorname{Rep}\left(\mathbb{Z}^{n}, P U(m)\right)\right)$.

The components $B_{n}(U(m))_{D}$ each give rise to a principal $\mathbb{T}^{n}$-bundle, and after dividing out by conjugation we see that the action of $\mathbb{T}^{n}$ is given by the simultaneous action on each unordered coordinate in the symmetric product through the homomorphism $\mathbb{T}^{n} \rightarrow \mathbb{T}^{n} / R(D)$; we denote these reduced symmetric products by $\widehat{S P^{\frac{m}{\sigma(D)}}}\left(\mathbb{T}^{n} / R(D)\right)$. Note that they are all in fact homeomorphic to the corresponding reduced symmetric product of $\mathbb{T}^{n}$. Combining the two results above we obtain

Theorem A.

$$
\operatorname{Rep}\left(\mathbb{Z}^{n}, P U(m)\right) \cong \coprod_{\substack{D \in T(n, \mathbb{Z} / m \mathbb{Z}) \\ \sigma(D) \mid m}} \widehat{S P^{\frac{m}{\sigma(D)}}}\left(\mathbb{T}^{n} / R(D)\right)
$$

The labelling of components can be understood using cohomology. As before we can identify $T(n, \mathbb{Z} / m \mathbb{Z})$ with $H^{2}\left(\mathbb{Z}^{n}, \mathbb{Z} / m \mathbb{Z}\right)$ using a basis. For a projective representation $h: \mathbb{Z}^{n} \rightarrow P U(m)$, there is a cohomology class in $H^{2}\left(\mathbb{Z}^{n}, \mathbb{Z} / m \mathbb{Z}\right)$ associated to it, defined as the pullback $\alpha=h^{*}(\nu)$, where $\nu \in H^{2}(B P U(m), \mathbb{Z} / m \mathbb{Z}) \cong \mathbb{Z} / m \mathbb{Z}$ is the canonical generator associated to $S U(m)$. The component corresponding to $h$ is precisely the one labelled by 
a skew symmetric matrix $D$ which represents $\alpha$. As this component is non-empty we must have $\sigma(\alpha) \mid m$.

Example 2.9. In the case when $n=2, H^{2}\left(\mathbb{Z}^{2}, \mathbb{Z} / m \mathbb{Z}\right) \cong \mathbb{Z} / m \mathbb{Z}$ and $\sigma(D)=|D|$, the order of $D$, which always divides $m$ and so $N(2, m)=m$. Our decomposition can be written as

$$
\operatorname{Rep}\left(\mathbb{Z}^{2}, P U(m)\right) \cong \widehat{S P^{m}}\left(\mathbb{T}^{2}\right) \bigsqcup \coprod_{\substack{D \in \mathbb{Z} / m \mathbb{Z} \\ 1<|D|<m}} \widehat{S P^{|m|}}\left(\mathbb{T}^{2} / R(D)\right) \bigsqcup\left\{x_{1}, \ldots, x_{\Phi(m)}\right\}
$$

where $\Phi$ is Euler's function (see [3], Proposition 9).

Example 2.10. We now consider the case when $m=p^{2}$ and $n \geq 4$. From the analysis in [1], section 3, we see that $\operatorname{Rep}\left(\mathbb{Z}^{n}, P U\left(p^{2}\right)\right)$ has

$$
N\left(n, p^{2}\right)=1+\frac{\left(p^{n-1}-1\right)\left(p^{n}-1\right)\left(p^{2 n+1}-p^{n}-p^{n-1}+p^{4}+p^{2}-1\right)}{\left(p^{2}-1\right)\left(p^{4}-1\right)}
$$

components, of which

$$
u(p)=\frac{\left(p^{n-1}-1\right)\left(p^{n}-1\right)}{p^{2}-1}
$$

correspond to $\sigma(D)=p$ and so we have

$$
\operatorname{Rep}\left(\mathbb{Z}^{n}, P U\left(p^{2}\right)\right) \cong \widehat{S P^{p}}\left(\mathbb{T}^{n}\right) \bigsqcup \coprod_{\frac{\left(p^{n-1}-1\right)\left(p^{n}-1\right)}{p^{2}-1}} \widehat{S P^{p}}\left(\mathbb{T}^{n} / R(D)\right) \bigsqcup\left\{x_{1}, \ldots, x_{N\left(n, p^{2}\right)-u(p)-1}\right\}
$$

Our analysis can be applied to describe the projective representations of any finitely generated abelian group. Let

$$
\Gamma=\mathbb{Z} / k_{1} \oplus \ldots \oplus \mathbb{Z} / k_{s} \oplus \mathbb{Z}^{n} \cong \operatorname{Tor}(\Gamma) \oplus \mathbb{Z}^{n}
$$

Define

$$
B(\Gamma, U(m))=\left\{\left(A_{1}, \ldots, A_{s+n}\right) \in B_{s+n}(U(m)): A_{i}^{k_{i}}=I_{m} \text { for } 1 \leq i \leq s\right\} .
$$

It is a subspace of $B_{s+n}(U(m))$ and is invariant under the action of the subgroup

$$
\left\{\left(\theta_{1}, \ldots, \theta_{s+n}\right): \theta_{i}^{k_{i}}=1 \text { for } 1 \leq i \leq s\right\} \cong \operatorname{Tor}(\Gamma) \times \mathbb{T}^{n} \subset \mathbb{T}^{s+n} .
$$

This group action commutes with the conjugation action of $U(m)$. Hence, $B(\Gamma, U(m))$ is a $U(m)$-equivariant, $\left(\operatorname{Tor}(\Gamma) \times \mathbb{T}^{n}\right)$-principal bundle over $\operatorname{Hom}(\Gamma, P U(m))$. Also, there is a decomposition of

$$
B(\Gamma, U(m))=\coprod B(\Gamma, U(m))_{D}
$$

into a disjoint union of subspaces indexed by $D \in T(s+n, \mathbb{Z} / m \mathbb{Z})$. The subspaces

$$
\operatorname{Hom}(\Gamma, P U(m))_{D} \subset \operatorname{Hom}(\Gamma, P U(m)) \text { and } \operatorname{Rep}(\Gamma, P U(m))_{D} \subset \operatorname{Rep}(\Gamma, P U(m))
$$

can be similarly defined.

Note that for $A_{i} \in U(m), A_{i}^{k_{i}}=I_{m}$ if and only if all the eigenvalues of $A_{i}$ are $k_{i}$-th roots of unity. By Theorem 2.6, we obtain the following results similar to Theorem 2.7 and Theorem A. 
ON THE MODULI SPACES OF COMMUTING ELEMENTS IN THE PROJECTIVE UNITARY GROUPS 7

Theorem 2.11. Let $\Gamma=\mathbb{Z} / k_{1} \oplus \ldots \oplus \mathbb{Z} / k_{s} \oplus \mathbb{Z}^{n} \cong \operatorname{Tor}(\Gamma) \oplus \mathbb{Z}^{n}$. Then

$$
B(\Gamma, U(m)) / U(m) \cong \coprod_{\substack{D \in T(s+n, \mathbb{Z} / m \mathbb{Z}) \\ \sigma(D) \mid m \text { and } r_{i}(D) \mid k_{i}}} S P^{\frac{m}{\sigma(D)}}\left(\left(\operatorname{Tor}(\Gamma) \times \mathbb{T}^{n}\right) / R(D)\right)
$$

and

$$
\operatorname{Rep}(\Gamma, P U(m)) \cong \coprod_{\substack{D \in T(s+n, \mathbb{Z} / m \mathbb{Z}) \\ \sigma(D) \mid m \text { and } r_{i}(D) \mid k_{i}}} \widehat{S P^{\frac{m}{\sigma(D)}}}\left(\left(\operatorname{Tor}(\Gamma) \times \mathbb{T}^{n}\right) / R(D)\right)
$$

Remark 2.12. Suppose $\sigma(D) \mid m$ and $r_{i}(D) \mid k_{i}$. Let $H$ be the cokernel of the composition $R(D) \hookrightarrow \operatorname{Tor}(\Gamma) \times \mathbb{T}^{n} \stackrel{\text { proj }}{\longrightarrow} \operatorname{Tor}(\Gamma)$ of inclusion followed by projection. Then, if we focus on the images of the components associated to $D$ under the quotient

$$
B(\Gamma, U(m)) \rightarrow \operatorname{Hom}(\Gamma, P U(m)),
$$

we have

$$
\pi_{0}\left(H \operatorname{om}(\Gamma, P U(m))_{D}\right) \cong \pi_{0}\left(\operatorname{Rep}(\Gamma, P U(m))_{D}\right) \cong \widehat{S P^{\frac{m}{\sigma(D)}}}(H) .
$$

The number of orbits on the right hand side can be computed using Burnside's lemma:

$$
\left|\widehat{S P^{\frac{m}{\sigma(D)}}}(H)\right|=\frac{1}{|H|} \sum_{g \in H}\left|S P^{\frac{m}{\sigma(D)}}(H)^{g}\right|
$$

where

$$
\left|S P^{\frac{m}{\sigma(D)}}(H)^{g}\right|= \begin{cases}\left(\begin{array}{cl}
\frac{m+|H| \sigma(D)}{|g| \sigma(D)}-1 \\
\frac{m}{|g| \sigma(D)}
\end{array}\right) & |g| \sigma(D) \mid m \\
0 & |g| \sigma(D) \nmid m\end{cases}
$$

Example 2.13 (Projective representations of finite abelian groups). Our results allow us to recover results about projective representations of finite abelian groups. Consider a finite abelian group $\Gamma=\mathbb{Z} / k_{1} \oplus \ldots \oplus \mathbb{Z} / k_{s}$, where $k_{i}$ divides $k_{i+1}$ for $i=1, \ldots, s-1$. By Theorem 2.11, the space of degree $m$ projective unitary representation modulo projective equivalence is given by

$$
\operatorname{Rep}(\Gamma, P U(m)) \cong \coprod_{\substack{D \in T(s, \mathbb{Z} / m \mathbb{Z}) \\ \sigma(D) \mid m \text { and } r_{i}(D) \mid k_{i}}} \widehat{S P^{\frac{m}{\sigma(D)}}}(\Gamma / R(D)) .
$$

If $\sigma(D)=m$, then $\widehat{S P^{\frac{m}{\sigma(D)}}}(\Gamma / R(D))$ is a single point and corresponds to an irreducible projective representation.

Removing the restriction on the dimension, we see that projective equivalence classes of irreducible projective representations of $\Gamma$ are in one-to-one correspondence with the $D \in T(n, \mathbb{Q} / \mathbb{Z})$ such that $r_{i}(D) \mid k_{i}$ for any $i=1, \ldots, s$. Note that since $D$ is skew-symmetric, the condition $r_{i}(D) \mid k_{i}$ is equivalent to $\left|d_{i j}\right|=\left|-d_{j i}\right|$ divides $k_{i}$ for $i<j$. 
In terms of cohomology, this indexing can be seen to arise from pulling back using the projection $p: \mathbb{Z}^{s} \rightarrow \Gamma$, which yields a factorization

$$
H^{2}(B P U(m), \mathbb{Z} / m \mathbb{Z}) \rightarrow H^{2}(\Gamma, \mathbb{Z} / m \mathbb{Z}) \rightarrow H^{2}\left(\mathbb{Z}^{s}, \mathbb{Z} / m \mathbb{Z}\right) .
$$

Note that $p^{*}: H^{2}(\Gamma, \mathbb{Q} / \mathbb{Z}) \rightarrow H^{2}\left(\mathbb{Z}^{s}, \mathbb{Q} / \mathbb{Z}\right)$ is injective, therefore if we consider all possible dimensions $m$ we see that the total indexing is in one-to-one correspondence with elements in $H^{2}(\Gamma, \mathbb{Q} / \mathbb{Z}) \cong H^{3}(\Gamma, \mathbb{Z})$. Moreover, for the projective equivalence class corresponding to $D$, its set of representatives, up to linear equivalence, is indexed by $\Gamma / R(D)$, and each such representation has degree $\sigma(D)$.

\section{Projective Representations and Flat Bundles}

We now reformulate the computation of path components using homotopy theory, following the approach in [4], Lemma 2.5. Recall that given a group homomorphism $h: \Gamma \rightarrow G$, it induces a continuous (pointed) map on classifying spaces $B h: B \Gamma \rightarrow B G$. For $G$ a compact connected Lie group, the correspondence

$$
\operatorname{Hom}(\Gamma, G) \rightarrow \operatorname{Map}_{*}(B \Gamma, B G)
$$

is continuous and so induces a map on path components. As conjugation by $G$ is homotopically trivial on $B G$, it gives rise to a map

$$
\pi_{0}(\operatorname{Rep}(\Gamma, G)) \rightarrow[B \Gamma, B G] .
$$

If $X$ is a path-connected CW-complex with $\pi_{1}(X)=\Gamma$, then composing with the classifying map $c: X \rightarrow B \pi_{1}(X)$ of the universal cover $\tilde{X} \rightarrow X$ we obtain a map

$$
\Psi_{X}^{G}: \pi_{0}\left(\operatorname{Rep}\left(\pi_{1}(X), G\right)\right) \rightarrow[X, B G]
$$

which measures the flat principal $G$-bundles on $X$.

In the case when $G=P U(m)$ we have a canonical homotopy class

$$
\Omega: B P U(m) \rightarrow K(\mathbb{Z} / m \mathbb{Z}, 2)
$$

associated to the central extension

$$
1 \rightarrow \mathbb{Z} / m \rightarrow S U(m) \rightarrow P U(m) \rightarrow 1
$$

For a CW-complex $X$, this gives rise to the composition

$$
\pi_{0}\left(\operatorname{Rep}\left(\pi_{1}(X), P U(m)\right)\right) \stackrel{\Psi_{X}^{P U(m)}}{\longrightarrow}[X, B P U(m)] \stackrel{\Omega}{\rightarrow}[X, K(\mathbb{Z} / m \mathbb{Z}, 2)]=H^{2}(X, \mathbb{Z} / m \mathbb{Z}) .
$$

We have

Theorem B. Let $X$ denote a connected $C W$-complex with $\pi_{1}(X)=\mathbb{Z}^{n}$; then the map $\Psi_{X}^{P U(m)}: \pi_{0}\left(\operatorname{Rep}\left(\pi_{1}(X), P U(m)\right) \rightarrow[X, B P U(m)]\right.$ is injective for all $n, m \geq 1$ and so there are $N(n, m)$ distinct isomorphism classes of flat principal $P U(m)$-bundles on $X$.

Proof. First we consider the basic case $X=B \mathbb{Z}^{n}=\mathbb{T}^{n}$ : the composition

$$
\pi_{0}\left(\operatorname{Rep}\left(\mathbb{Z}^{n}, P U(m)\right)\right) \stackrel{\Psi_{\mathbb{T}^{n}}^{P U(m)}}{\longrightarrow}\left[\mathbb{T}^{n}, B P U(m)\right] \stackrel{\Omega}{\rightarrow}\left[\mathbb{T}^{n}, K(\mathbb{Z} / m \mathbb{Z}, 2)\right]=H^{2}\left(B \mathbb{Z}^{n}, \mathbb{Z} / m \mathbb{Z}\right)
$$


ON THE MODULI SPACES OF COMMUTING ELEMENTS IN THE PROJECTIVE UNITARY GROUPS 9

is the map on path components described in Section 2. We know that its image has precisely $N(n, m)$ elements, corresponding to the skew symmetric matrices $D$ with $\sigma(D)$ dividing $m$. In other words, this map distinguishes components, and is injective. If

$$
c^{*}: H^{2}\left(B \mathbb{Z}^{n}, \mathbb{Z} / m \mathbb{Z}\right) \rightarrow H^{2}(X, \mathbb{Z} / m \mathbb{Z})
$$

is induced by the classifying map, by naturality we have

$$
c^{*} \circ \Omega \circ \Psi_{\mathbb{T}^{n}}^{P U(m)}=\Omega \circ \Psi_{X}^{P U(m)} .
$$

From the five-term exact sequence in cohomology for the fibration $\tilde{X} \rightarrow X \rightarrow B \pi_{1} X$ we infer that $c^{*}: H^{2}\left(B \pi_{1}(X), \mathbb{Z} / m \mathbb{Z}\right) \rightarrow H^{2}(X, \mathbb{Z} / m \mathbb{Z})$ is in fact injective. Therefore the composition $\Omega \circ \Psi_{X}^{P U(m)}$ is injective and so is $\Psi_{X}^{P U(m)}$.

Next we study the surjectivity of the map

$$
\Psi_{\mathbb{T}^{n}}^{P U(m)}: \pi_{0}\left(\operatorname{Rep}\left(\mathbb{Z}^{n}, P U(m)\right) \rightarrow\left[\mathbb{T}^{n}, B P U(m)\right] .\right.
$$

We begin with the following lemma

Lemma 3.1. Let $m \geq 2$. If $n \leq 3$, $\left[\mathbb{T}^{n}, B P U(m)\right]$ is finite, of cardinality equal to that of $T(n, \mathbb{Z} / m \mathbb{Z})$. If $n \geq 4$, the set $\left[\mathbb{T}^{n}, B P U(m)\right]$ has infinitely many elements.

Proof. This follows from Woodward's classification of principal $P U(m)$-bundles for low dimensional complexes (see [9], page 514). For $\mathbb{T}^{n}, n \leq 3$ he shows that the map

$$
\left[\mathbb{T}^{n}, B P U(m)\right] \rightarrow H^{2}\left(\mathbb{T}^{n}, \mathbb{Z} / m \mathbb{Z}\right) \cong T(n, \mathbb{Z} / m \mathbb{Z})
$$

is a bijection. We outline a direct proof that $\left[\mathbb{T}^{n}, B P U(m)\right]$ must be infinite for $n \geq 4$. For $n=4$, there is a map $\Phi: \mathbb{T}^{4} \rightarrow \mathbb{S}^{4}$ which induces an isomorphism on $H_{4}$. This arises from using the 4-dimensional cell in a CW-complex decomposition for $\mathbb{T}^{4}$ from its structure as a product of circles, each having a single 0-cell and a single 1-cell. The map $B S U(m) \rightarrow B P U(m)$ induces an isomorphism $\mathbb{Z}=\pi_{4}(B S U(m)) \cong \pi_{4}(B P U(m))$ and so it is possible to choose a map $\rho: \mathbb{S}^{4} \rightarrow B P U(m)$ realizing this isomorphism. The Hurewicz map $\pi_{4}(B P U(m)) \rightarrow H_{4}(B P U(m), \mathbb{Z})$ can be identified with the monomorphism $H_{4}(B S U(m), \mathbb{Z}) \rightarrow H_{4}(B P U(m), \mathbb{Z})$, hence the composition $\rho \circ \Phi: \mathbb{T}^{4} \rightarrow B P U(m)$ is a map inducing an injection on the fundamental class in $\mathbb{T}^{4}$. It follows that $\left[\mathbb{T}^{4}, B P U(m)\right]$ cannot be finite. For $n \geq 4$, we can use the split surjection $\left[\mathbb{T}^{n}, B P U(m)\right] \rightarrow\left[\mathbb{T}^{4}, B P U(m)\right]$ to verify the claim.

Proposition 3.2. Let $m \geq 2$, then $\Psi_{\mathbb{T}^{n}}^{P U(m)}: \pi_{0}\left(\operatorname{Rep}\left(\mathbb{Z}^{n}, P U(m)\right) \rightarrow\left[\mathbb{T}^{n}, B P U(m)\right]\right.$ is surjective if and only if $n \leq 3$.

Proof. From the definition in cohomology (and using an appropriate basis when $n=3$ ) it is easy to see that for $n=1,2,3$, we have that $\{D \in T(n, \mathbb{Z} / m \mathbb{Z}): \sigma(D) \mid m\}=T(n, \mathbb{Z} / m \mathbb{Z})$. Thus we conclude that $\Omega \circ \Psi_{\mathbb{T}^{n}}^{P U(m)}$ is a bijection and therefore by a cardinality argument so is $\Psi_{\mathbb{T}^{n}}^{P U(m)}$ for $n=1,2,3$. For $n \geq 4$ we have verified that $\left[\mathbb{T}^{n}, B P U(m)\right]$ is infinite, whence the result follows.

Corollary 3.3. There exists a principal PU(m)-bundle on the $n$-torus $\mathbb{T}^{n}$ which does not admit a flat structure if and only if $n \geq 4$. 


\section{ACKNOWLEDGEMENTS}

The first author was funded by NSERC. We are grateful to M. Bergeron, J.M. Gómez, Z. Reichstein and B. Williams for their helpful comments.

\section{REFERENCES}

[1] Alejandro Adem and Man Chuen Cheng. Representation spaces for central extensions and almost commuting unitary matrices. J. London Math. Soc. 94 (2): pp 503-524 (2016).

[2] Alejandro Adem, Frederick R. Cohen, and José Manuel Gómez. Stable splittings, spaces of representations and almost commuting elements in Lie groups Math. Proc. Camb. Phil. Soc., 149, 455-490, 2010.

[3] Alejandro Adem, Frederick R. Cohen, and José Manuel Gómez. Commuting elements in central products of special unitary groups Proc. Edinburgh Math. Soc. (Series 2) 56(1):1-12, 2013.

[4] Alejandro Adem and Frederick R. Cohen. Commuting elements and spaces of homomorphisms. Math. Ann., 338(3):587-626, 2007.

[5] Maxime Bergeron. The topology of nilpotent representations in reductive groups and their maximal compact subgroups. Geom. Topol., 19(3):1383-1407, 2015.

[6] Armand Borel. Sous-groupes commutatifs et torsion des groupes de Lie compacts connexes. Tohoku Math. J., (2) Vol. 13, Number 2 (1961), 216-240.

[7] Armand Borel, Robert Friedman, and John W. Morgan. Almost commuting elements in compact Lie groups. Mem. Amer. Math. Soc., 157(747):x+136, 2002.

[8] Zinovy Reichstein and Nikolaus Vonessen. Rational central simple algebras. Israel J. Math., 95:253-280, 1996.

[9] L. M. Woodward The Classification of principal PU(n)-bundles over a 4-complex J. London Math. Soc., (2), 25 (1982), 513-524.

Department of Mathematics, University of British Columbia, Vancouver BC V6T 122 , CANADA

E-mail address: adem@math.ubc.ca

Department of Mathematics, The Chinese University of Hong Kong, Shatin, N.T., Hong KONG

E-mail address: mccheng@math.cuhk.edu.hk 